\title{
The coupling finite-boundary element method for soil-structure interaction under spatially varying ground motion
}

\author{
Z. Tonyali ${ }^{1}$, S. Ates ${ }^{* 2}$ \\ ${ }^{1}$ Recep Tayyip Erdoğan University, Department of Civil Engineering, 53100, Rize, Turkey \\ ${ }^{2}$ Karadeniz Technical University, Department of Civil Engineering, 61080, Trabzon, Turkey
}

\begin{abstract}
In this study, a coupled model based on the boundary element and the finite element methods is used to analyze the dynamic responses of two-dimensional model resting on layered soil medium under spatially varying ground motion effects. The dynamic response of the soil-structure systems is obtained in the frequency domain. The results of the finite element and the coupling finite-boundary element models are compared with each local soil conditions. In the seismic analysis of the system, the substructure method is employed. In the standard finite element model, both the structure and semi-infinitive soil medium are modeled by the finite elements, however, in the coupling finite-boundary element model, the structure and the soil medium are modeled respectively by the finite and boundary elements, both. In the last method, the special features and advantages of two methodologies are considered. In the coupling of finite-boundary element method, the equivalent finite element approach is used in which the boundary element region is transformed as an equivalent finite element and the final system is solved as a stiffness problem. Results of the study show that the coupling finite-boundary element method can provide realistic and effective modeling of soil-structure interaction problems as compared with standard finite element method results.
\end{abstract}

\section{Keywords}

Boundary element method; Coupling finite-boundary element method; Finite element method; Soil-structure interaction; Spatially varying ground motion

Received: 16 March 2018; Accepted: 29 March 2018

ISSN: 2630-5763 (online) C 2018 Golden Light Publishing® All rights reserved.

\section{Introduction}

One of the significant problems in geotechnical engineering is to determine the response of structures together with the surrounding soils. Therefore, numerical model of the system should consider soil-structure interaction effects in the analysis and design of structures founded on soil medium. Now, the finite element method (FEM) is undoubtedly the most popular and useful technique for modeling of structures. Boundary element method (BEM) is considered as one of the most powerful alternative of the former numerical method and at the same time it is the most appropriate technique due to seismic wave propagation in a complex geological media. These methods can be formulated either in time or in frequency domain, and each of these methods has its own advantages and drawbacks. The good thing is that each drawback of one of the both methods should be overcome by an advantage of the another. The decision of which methods should be employed in the modeling and analyzing of 
structure depends on the properties of the system such as geometry, material properties, type of loading and boundary conditions. To profit from the advantages of each of two methods and by evading their respective disadvantages, it seemed to be quite promising to develop combined formulations. Therefore, in today's technology a combined model of the finite element and the boundary element methods is advantageously employed in numerical modeling of the infinite or semi-infinite domains, material non-linearity and region of high stress concentrations where finite and boundary elements are not particularly suitable on their own.

The finite element method is highly convenient for non-homogeneous and anisotropic materials as well as for dealing with the nonlinear behavior of a body. In the finite element method for infinite or semi-infinite regions, an extensive mesh to model the surrounding soil medium, namely near-field, is required and far-field is represented by artificial boundaries. Artificial boundaries introduce spurious reflections, which contaminate the numerical solutions in numerical modeling of wave propagation problems. However, the use of the boundary element method is more advantageous for systems with infinite extension and regions of high stress concentration.

White et al. [1] developed special boundary conditions called non-reflecting viscous boundary conditions that absorb the wave energy. However, the use of the boundary element method is by far more favorable than the non-reflecting viscous boundary conditions for systems with infinite extension and regions of high stress concentration about other methods [2]. The scaled boundaryfinite element method is an alternative and effective method for modeling systems with finite and infinite extension having non-homogeneous and incompressible material properties [3]. More details are given, e.g., by Hughes [4], Wolf and Song [5] and Bathe [6] for the finite element method and by Beskos [7], Becker [8] or Dominguez [9] for the boundary element method.

Zienkiewicz et al. [10] were among the first authors who proposed the coupling of finite and boundary elements. Cruse and Wilson [11] used a coupled finite-boundary element formulation for the solution of two-dimensional elastic fracture problems.

Brebbia and Georgiou [12] proposed two different approaches to couple the finite and boundary elements in two dimensional elastic problems. They used two different approaches to couple the finite and boundary elements. The first one treats the boundary element region as a macro finite element. This leads to a nonsymmetrical stiffness matrix for boundary element region. To prevent this hardship, they proposed simple and effective way such as the least squares technique. The second one treats the finite element region as an equivalent boundary element region. They studied numerical aspects of two alternative approaches for two dimensional elastic problems. Brady and Wassyng [13] used the coupled finiteboundary element technique to determine stress and displacement distributions in a rock structure caused by underground excavation. Tullberg and Bolteus [14] developed different boundary element stiffness matrices for two-dimensional elasticity problems and seriously studied to choose the best one for the coupling of FEM and BEM. It was concluded that the direct non-symmetric stiffness matrix is the best one and the direct non-symmetric stiffness matrix is as good as, or better then finite element stiffness matrix. Beer [15] employed the details of application of boundary elements into an existing finite element software. A computer code was examined, and comparison was made between the finite element, the boundary element and the coupled method as applied to unbounded problems in two-dimensional elasticity and plasticity. Beer and Swoboda [16] used a combined finite-boundary element method for the elasto-plastic analysis of shallow tunnels in plain strain. Based on the principle of stationary potential energy, the equivalent boundary element stiffness matrix was given, and it was assembled with the finite element stiffness matrix in the usual way. Mitsui et al [17] presented coupling scheme for the boundary and finite elements using a joint element of Goodman in the analysis of several footings. It was obtained 
that coupling method is better than FEM for a structure founded on a multi-layer. Subei et al. [18] employed a combined finite-boundary element technique for the analysis of distresses in pavements subjected to mechanical and environmental effects. The non-homogeneity and the irregular geometry at the pavement joints are modeled by means of the finite elements, while the boundary elements are used to model the far field region at infinity. Yazdchi et al. [19] presented a study on the transient response of an elastic structure embedded in an elastic half-plane. The coupled finite-boundary element technique was employed, and transient dynamic and seismic forces were considered in the analysis. The finite element method was used for discretization of the near field, containing structures and its surrounding soil and the boundary element method was used to model the semi-infinite far field. These two methods were coupled through the equilibrium and compatibility conditions at the soil-structure interface. As an application of the proposed formulation, a gravity dam has been analyzed and the results for different foundation stiffness are presented. Von Estorff and Firuziaan [20] presented a general coupled boundary elementfinite element formulation for the investigation of dynamic soil-structure interaction including nonlinearities. The structure itself and the surrounding soil in the near field were modeled with finite elements, whereas the remaining soil region is modeled with the boundary elements. Thus, wave radiation to infinity was considered in the model. It was shown that the method is numerically powerful and can be used effectively for the nonlinear analyses of complex soil-structure interaction problems. Chouw and Hao [21] investigated the influence of the spatially varying ground excitations and soil-structure interaction on the pounding potential of two adjacent bridge frames. The bridge frames with their foundations and the subsoil were described by using a combined finite element and boundary element method. The spatially varying ground excitations were simulated stochastically based on the Japanese design spectrum for soft and medium soil. Genes and
Kocak [22] presented a coupled model based on finite-boundary element methods and scaled boundary finite element method for dynamic response of two dimensional structures embedded in layered soil media. Boundary element method was used for modeling the dynamic response of the unbounded media (far-field), whereas the standard finite element method was used for modeling the finite region (near-field) and the structure. Dynamic responses of rigid and elastic structures belong to the proposed model are investigated. It was obtained that the results of the study are in accordance with the results presented in the literature for the chosen problems.

A three-dimensional finite element model with viscous boundary was developed to investigate the seismic response of the cantilever wall by Cakir [23]. Yaseri et al. [24] studied the application of the scaled boundary finite-element method to the threedimensional analysis of ground vibrations induced by underground trains. The surrounding medium around the tunnel was simulated by the scaled boundary finite element method. The track was modeled with the finite-element method. It was observed that the method is an accurate approach for modeling the large-scale dynamic problems in engineering practice such as underground traininduced ground vibrations.

In the present paper, a finite element model and a coupled model based on boundary element and finite element methods are used to analyze the dynamic responses of two-dimensional model resting on layered soil medium under spatially varying ground motion effects and the results obtained are compared. In the finite element model, the structure itself and layered soil medium are modeled with the finite elements. In the coupled model, the structure itself is modeled with the finite elements, whereas the layered soil medium is modeled with the boundary elements. In the last model, these two methods are coupled through the equilibrium and compatibility conditions at the soil-structure interface. Using this procedure, the boundary element region is condensed on the interface of finite and boundary element regions and the size of the final system of equations is 
determined from only the finite element region only. In the coupled model, equivalent finite element approach is used in which boundary element region is transformed as an equivalent finite element and the final system is solved as a stiffness problem. The dynamic response of the soil-structure systems subjected to spatially varying ground motion is obtained in the frequency domain. In the seismic analysis of the system, the substructure method is employed to deal with the interaction problem. It has been shown that the similar results can be achieved with a numerical model in validating the developed coupled finiteboundary element method against the classical finite element method.

\section{The finite element and boundary element methods}

The finite element and the boundary element methods have become the two dominant numerical methods for solving science and engineering problems. The finite element method has been long established and is most well known in different fields of engineering problems. Thereafter, the boundary element method appeared offering new computational capabilities with its effectiveness, accuracy and low computational cost. The general approach in the finite element method is that the whole domain is subdivided into many finite elements joined together at some points called nodes. When the method is used for a solution of static problems, we get a set of simultaneous linear equations finally, which can be stated in the form

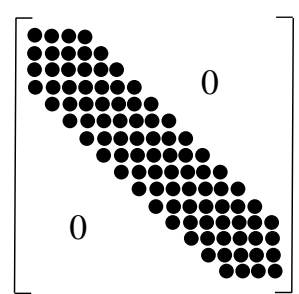

FEM

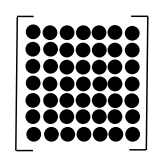

BEM
Fig. 1. Coefficient matrices for FEM and BEM
$\mathrm{Ku}=\mathrm{f}$

where $\mathrm{K}$ is the stiffness matrix of a structure, $\mathrm{u}$ is the displacement vector and $\mathrm{f}$ are the force vector. In a FEM model, the coefficient matrices have a great form since they are banded and symmetric. However, in a BEM model they are fully populated and non-symmetric. In addition, the matrices are generally of different sizes due to the differences in size of the domain mesh (FEM) compared to the surface mesh (BEM). This disadvantage of the BEM is counterbalanced by the much smaller dimensions of its matrices than FEM model. The general format of the coefficient matrices for a FEM and BEM model is given in Fig. 1.

The BEM model reduces the spatial dimension of the problem by one since only the boundaries of the domain are needed to be discretized as shown in Fig. 2. In addition, the data preparation and modeling time reduce especially for three dimensional problems having complex geometries as compared to FEM model. If the region is extended to infinity no artificial boundaries are required in the BEM, whereas those are required in the other methods such as finite difference and finite element. Therefore, BEM is very attractive for the treatment of linear problems defined in both bounded and unbounded domains.

The final expression of FEM has a relationship displacement at all nodes to nodal forces, whereas the final expression of BEM has a relationship between nodal displacements and nodal tractions. In BEM formulation, the dynamic response of homogeneous, isotropic and linear elastic structures in frequency domain, is described by the system equation as

$\mathrm{Hu}=\mathrm{G} \mathrm{t}$

where $\mathrm{H}$ and $\mathrm{G}$ are the system matrices obtained by the integration of the first and second fundamental solutions of BEM over the elements. The $u$ and $t$ are the displacement and the traction vectors, respectively. 


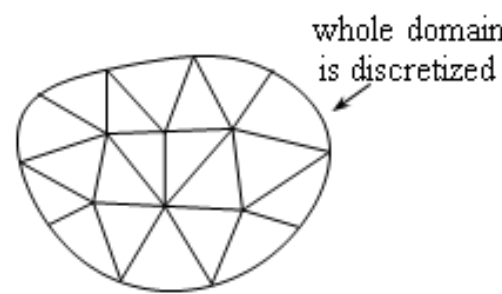

FEM

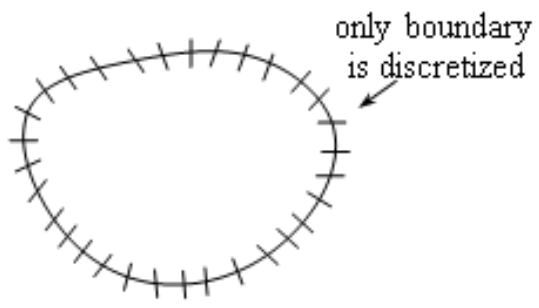

BEM

Fig. 2. Discretization of the domain for FEM and BEM

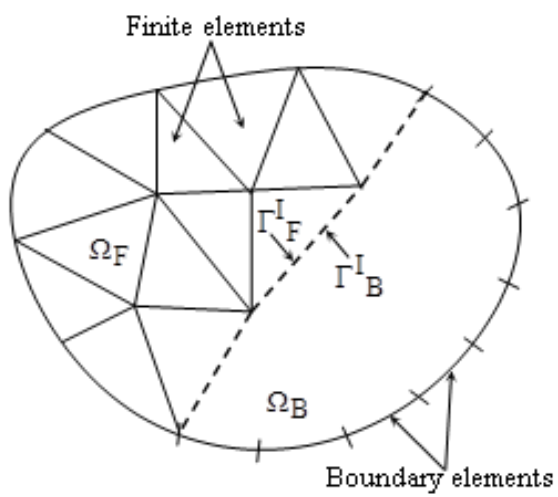

Fig. 3. The domain divided into finite element and boundary element regions

\section{Coupling finite-boundary element method}

This part explains the coupling finite-boundary element method. There are basically two different approaches to couple finite element and boundary element methods. The first one is equivalent finite element approach in which the boundary element region is treated as a large finite element and its stiffness is computed and assembled into the global stiffness matrix. The second one is equivalent boundary element approach in which the finite element region are treated as an equivalent boundary element region and their stiffness matrix is determined and assembled [12, 24]. The choice of coupling method depends mainly on the software available for the implementation, i.e., if boundary element capabilities are to be added to a finite element program, or finite element capabilities to a boundary element one. In this study, the equivalent finite element approach is used for analysis of response of structures to spatially varying ground motion, including the effects of structure-soil interaction and the substructure method is employed in which the unbounded soil is modeled by BEM and the structure is modeled by a standard FEM.

In the coupling technique, whole domain is divided into two regions $\Omega_{F}$ and $\Omega_{B}$ with common interface indicated by dotted line in Fig. 3. In the figure, $\Omega_{F}$ and $\Omega_{B}$ represent finite element and boundary element domains, respectively, whereas $\Gamma_{F}^{I}$ and $\Gamma_{B}^{I}$ represent boundaries for common interface domains $\Omega_{F}$ and $\Omega_{B}$. To ensure a correct coupling between finite element and boundary element meshes, the conditions of equilibrium and compatibility must be satisfied at the connecting interface. Therefore, coupling the two domains at the interface is provided using the displacement compatibility and the dynamic equilibrium equations at the soilstructure interface elements.

Satisfaction of compatibility conditions gives the same $u_{F}^{I}$ and $u_{B}^{I}$ interface displacements, i.e.

$\mathrm{u}_{\mathrm{F}}^{\mathrm{I}}=\mathrm{u}_{\mathrm{B}}^{\mathrm{I}}$ 
and the satisfaction of equilibrium conditions gives zero sum of forces along the interface, i.e.

$\mathrm{f}_{\mathrm{F}}^{\mathrm{I}}+\mathrm{f}_{\mathrm{B}}^{\mathrm{I}}=0$

In case of equivalent FEM, for $\Omega_{B}$ region, the boundary element stiffness matrix is obtained, which is summarized with the finite elements of the domain $\Omega_{F} \quad[12,14]$. In Fig 4, it is comprehensively demonstrated how to obtain the equivalent stiffness matrix belong to the boundary element region. In the figure, the system of equations of BEM is simulated to the system of equations of FEM. $\left[\mathrm{K}_{B}\right]$ and $\left\{\mathrm{f}_{B}\right\}$ are, respectively, boundary element stiffness matrix and nodal force vector for domain $\Omega_{B}$. [M] is the distribution matrix and it is interconnected to nodal tractions with the nodal forces as can be seen in Eq. (5). The general expression of transformation matrix is defined by Brebbia et al. (1984), i.e.

$\mathrm{M}=\int_{\Gamma} \mathrm{N}^{\mathrm{T}} \mathrm{N} \mathrm{d} \Gamma$

The coefficients of $\mathbf{M}$ depend on the type of interpolation functions, denoted by $\mathrm{N}$ in Eq. (5). For whole region, $\Omega_{F}+\Omega_{B}$ final system equation is given by Brebbia et al. [25] as

$$
\mathrm{K}^{\prime} \mathrm{u}=\mathrm{f}^{\prime}
$$

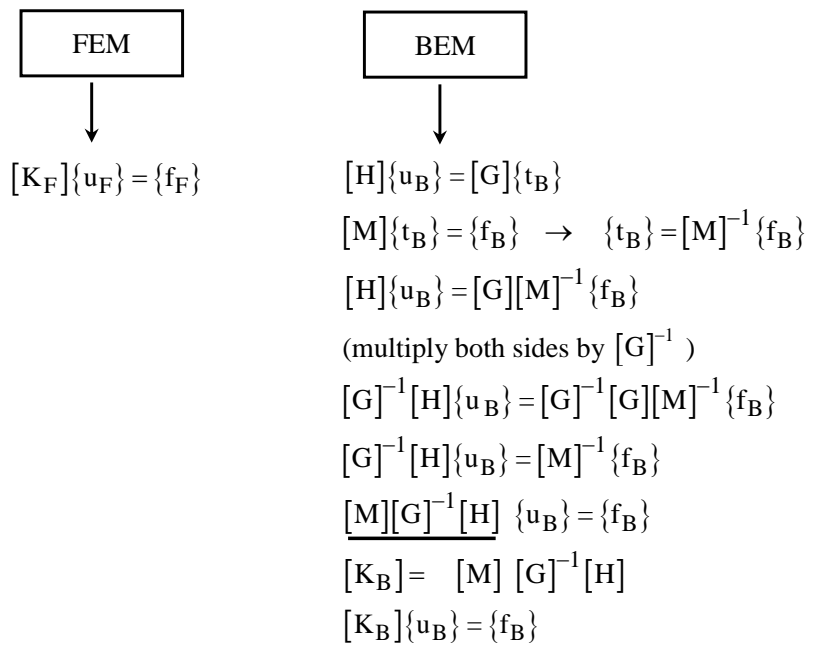

Fig. 4. Demonstration of obtaining equivalent finite element stiffness matrix for boundary element region

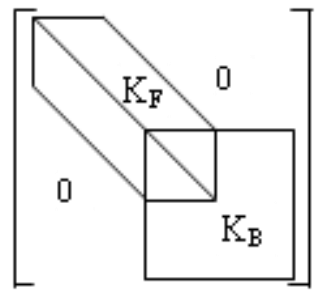

Fig. 5. General representation of coupled finite-boundary global stiffness matrix 
where

$$
\begin{aligned}
& K^{\prime}=\mathrm{K}_{\mathrm{F}}+\mathrm{K}_{\mathrm{B}} \\
& \mathrm{f}^{\prime}=\mathrm{f}_{\mathrm{F}}+\mathrm{f}_{\mathrm{B}}
\end{aligned}
$$

$\mathrm{K}_{\mathrm{F}}$ and $\mathrm{f}_{\mathrm{F}}$ are respectively global stiffness matrix and nodal force vector for finite element domain $\Omega_{F} . \mathrm{K}^{\prime}$ and $\mathrm{f}^{\prime}$ are respectively final global stiffness matrix of coupled finite-boundary elements and nodal force vector for whole domain $\Omega_{F}+\Omega_{B}$. In general, $\mathrm{K}^{\prime}$ is not symmetric stiffness matrix because stiffness matrix obtained $\mathrm{K}_{\mathrm{B}}$ for the boundary element domain is not symmetric. This makes it mostly inconvenient to use with the symmetric finite element global stiffness matrix. In this study, the procedure is used to make symmetric the boundary element stiffness matrix suggested by Brebbia et al [25].

In case of bounded problems, on the one hand, the banded matrix from the boundary element region and on the other hand the fully populated matrix from the boundary element region are coupled which schematically is shown in Fig. 5. It is clear from the figure that the size of bandwidth of the global matrix depends on the size of boundary element region and increases with the increase of its size which makes the coupled method less effective for these kinds of problems.

To avoid the above difficulty and make the coupled method effective in this work, the static condensation procedure is used which reduces the boundary element stiffness matrix along the interface and the size of the problem can be determined from degrees of freedom of the FE region as in the case of the unbounded problem. The formulation of this procedure is given in the following.

In presentation simplicity of the procedure, the problem shown in Fig. $3, \Omega_{F}$ finite and $\Omega_{B}$ boundary element regions are joined by an interface $\Gamma$. Hereby, $\Omega_{F}$ finite region is divided into finite elements, whereas $\Omega_{B}$ boundary region is divided into boundary elements. For $\Omega_{F}$ region, Eq. (1) can be rewritten in the form,
$\left[\begin{array}{ll}\mathrm{K}_{\mathrm{FF}} & \mathrm{K}_{\mathrm{FI}} \\ \mathrm{K}_{\mathrm{IF}} & \mathrm{K}_{\mathrm{II}}\end{array}\right]\left\{\begin{array}{l}\mathrm{u}_{\mathrm{F}} \\ \mathrm{u}_{\mathrm{I}}\end{array}\right\}=\left\{\begin{array}{l}\mathrm{f}_{\mathrm{F}} \\ \mathrm{f}_{\mathrm{IF}}\end{array}\right\}$

and for $\Omega_{B}$ region Eq. (2) can be rewritten in the form,

$$
\left[\begin{array}{ll}
\mathrm{H}_{\mathrm{II}} & \mathrm{H}_{\mathrm{IB}} \\
\mathrm{H}_{\mathrm{BI}} & \mathrm{H}_{\mathrm{BB}}
\end{array}\right]\left\{\begin{array}{l}
\mathrm{u}_{\mathrm{I}} \\
\mathrm{u}_{\mathrm{B}}
\end{array}\right\}=\left[\begin{array}{ll}
\mathrm{G}_{\mathrm{II}} & \mathrm{G}_{\mathrm{IB}} \\
\mathrm{G}_{\mathrm{BI}} & \mathrm{G}_{\mathrm{BB}}
\end{array}\right]\left\{\begin{array}{c}
\mathrm{t}_{\mathrm{IB}} \\
\mathrm{t}_{\mathrm{B}}
\end{array}\right\}
$$

In Eq. (8) and Eq. (9), subscripts IB and IF etc. denotes interface-finite, boundary-interface and so on. In order to obtain equivalent FEM expression from Eq. (9), the formulation for the equivalent FEM demonstrated in Fig. 4 is used. For boundary region, final equivalent FEM expression is

$\left[\begin{array}{ll}\mathrm{K}_{\mathrm{II}} & \mathrm{K}_{\mathrm{IB}} \\ \mathrm{K}_{\mathrm{BI}} & \mathrm{K}_{\mathrm{BB}}\end{array}\right]\left\{\begin{array}{l}\mathrm{u}_{\mathrm{I}} \\ \mathrm{u}_{\mathrm{B}}\end{array}\right\}=\left\{\begin{array}{c}\mathrm{f}_{\mathrm{IB}} \\ \mathrm{f}_{\mathrm{B}}\end{array}\right\}$

From Eq. (10),

$\mathrm{u}_{\mathrm{B}}=\left(\mathrm{K}_{\mathrm{BB}}\right)^{-1}\left[\mathrm{f}_{\mathrm{B}}-\mathrm{K}_{\mathrm{BI}} \mathrm{u}_{\mathrm{I}}\right]$

obtained and substituting this into part of the same equation

$\left[\mathrm{K}_{\mathrm{II}}-\mathrm{K}_{\mathrm{IB}}\left(\mathrm{K}_{\mathrm{BB}}\right)^{-1} \mathrm{~K}_{\mathrm{BI}}\right] \mathrm{u}_{\mathrm{I}}=\mathrm{f}_{\mathrm{IB}}-\mathrm{K}_{\mathrm{IB}}\left(\mathrm{K}_{\mathrm{BB}}\right)^{-1} \mathrm{f}_{\mathrm{B}}$

which can be reduced to

$\mathrm{K}_{\mathrm{B}}^{\mathrm{R}} \mathrm{u}_{\mathrm{I}}=\mathrm{f}_{\mathrm{IB}}-\mathrm{f}_{\mathrm{B}}^{\mathrm{R}}$

where $\mathrm{K}_{\mathrm{B}}^{\mathrm{R}}$ is a condensed boundary element stiffness matrix on the interface $\Gamma^{I}$ and $\mathrm{f}_{\mathrm{B}}^{\mathrm{R}}$ is the condensed force vector.

To combine $\Omega_{F}$ and $\Omega_{B}$ regions Eq. (3) compatibility and Eq. (4) equilibrium conditions must be satisfied on $\Gamma$ interface. In the present case,

$$
\begin{aligned}
& \mathrm{u}_{\mathrm{IF}}=\mathrm{u}_{\mathrm{IB}}=\mathrm{u}_{\mathrm{I}} \\
& \mathrm{f}_{\mathrm{IF}}=-\mathrm{f}_{\mathrm{IB}}
\end{aligned}
$$

For the whole region $\Omega=\Omega_{F}+\Omega_{B}$, the final expression can be obtained from Eq. (8), Eq. (13), and Eq. (14) 


$$
\left[\begin{array}{cc}
\mathrm{K}_{\mathrm{FF}} & \mathrm{K}_{\mathrm{FI}} \\
\mathrm{K}_{\mathrm{IF}} & \mathrm{K}_{\mathrm{II}}+\mathrm{K}_{B}^{R}
\end{array}\right]\left\{\begin{array}{l}
\mathrm{u}_{\mathrm{F}} \\
\mathrm{u}_{\mathrm{I}}
\end{array}\right\}=\left\{\begin{array}{c}
\mathrm{f}_{\mathrm{F}} \\
-\mathrm{f}_{B}^{R}
\end{array}\right\}
$$

From Eq. (15), unknown $u_{F}$ and $u_{I}$ displacements can be determined and then for region displacements can be received from Eq. (11). As can be seen from Eq. (15) the final number of equations is determined only from degrees of freedom of the finite element domain.

\section{Spatially varying ground motion}

The equation of motion of a structural system can be written as

$$
[\mathrm{M}]\{\ddot{\mathrm{v}}\}+[\mathrm{C}]\{\dot{\mathrm{v}}\}+[\mathrm{K}]\{\mathrm{v}\}=\{\mathrm{F}\}
$$

where $[\mathrm{M}],[\mathrm{C}]$ and $[\mathrm{K}]$ are the mass, damping and stiffness matrices, respectively; $\{\ddot{v}\},\{\dot{v}\}$ and $\{v\}$ are vectors of total accelerations, velocities and displacements, respectively and $\{\mathrm{F}\}$ is a vector of input forces.

The degrees of freedom can be defined as known and unknown. The known degrees of freedom are associated with those of the structurefoundation interface. The unknowns are related to degrees of freedom of the structure. The former degrees of freedom will be denoted hereafter as the vector $v_{g}$, and the latter as $v_{r}$. Here, the subscript ' $g$ ' denotes the ground degrees of freedom and ' $r$ ' denotes the response degrees of freedom. Eq. (16) can be rearranged by separating the degrees of freedom into two groups as known and unknown [26-28]

$$
\begin{array}{r}
{\left[\begin{array}{cc}
\mathrm{M}_{\mathrm{rr}} & \mathrm{M}_{\mathrm{rg}} \\
\mathrm{M}_{\mathrm{gr}} & \mathrm{M}_{\mathrm{gg}}
\end{array}\right]\left\{\begin{array}{c}
\ddot{\mathrm{v}}_{\mathrm{r}} \\
\ddot{\mathrm{v}}_{\mathrm{g}}
\end{array}\right\}+\left[\begin{array}{cc}
\mathrm{C}_{\mathrm{rr}} & \mathrm{C}_{\mathrm{rg}} \\
\mathrm{C}_{\mathrm{gr}} & \mathrm{C}_{\mathrm{gg}}
\end{array}\right]\left\{\begin{array}{c}
\dot{\mathrm{v}}_{\mathrm{r}} \\
\dot{\mathrm{v}}_{\mathrm{g}}
\end{array}\right\}} \\
+\left[\begin{array}{cc}
\mathrm{K}_{\mathrm{rr}} & \mathrm{K}_{\mathrm{rg}} \\
\mathrm{K}_{\mathrm{gr}} & \mathrm{K}_{\mathrm{gg}}
\end{array}\right]\left\{\begin{array}{c}
\mathrm{v}_{\mathrm{r}} \\
\mathrm{v}_{\mathrm{g}}
\end{array}\right\}=\left\{\begin{array}{l}
0 \\
0
\end{array}\right\}
\end{array}
$$

It is possible to separate the total displacement vectors as quasi-static and dynamic components as follows: $\left\{\begin{array}{c}\mathrm{v}_{\mathrm{r}} \\ \mathrm{v}_{\mathrm{g}}\end{array}\right\}=\left\{\begin{array}{c}\mathrm{v}_{\mathrm{sr}} \\ \mathrm{v}_{\mathrm{sg}}\end{array}\right\}+\left\{\begin{array}{c}\mathrm{v}_{\mathrm{dr}} \\ \mathrm{v}_{\mathrm{dg}}\end{array}\right\}$

Because of complex nature of the earth crust, earthquake ground motions will not be the same at distances of the dimensions of long span structure. While analyzing large structures, spatially varying earthquake ground motions should be considered, and total displacements have to be used in expressing the governing equation of motion. The spatially varying earthquake ground motion includes incoherence, wave-passage and siteresponse effects. These effects are characterized by the coherency function in frequency domain.

The spatial variability of the ground motion is characterized by the coherency function $\gamma_{1 \mathrm{~m}}(\omega)$. The cross-power spectral density function between the accelerations $\ddot{\mathrm{v}}_{g_{l}}$ and $\ddot{\mathrm{v}}_{g_{m}}$ at the support points 1 and $\mathrm{m}$ is written as [29 30];

$$
S_{\dot{v}_{g_{l}}, \ddot{v}_{g_{m}}}(\omega)=\gamma_{l m}(\omega) \sqrt{S_{\ddot{v}_{g_{l}} \ddot{v}_{g_{l}}}(\omega) S_{\ddot{v}_{g_{m}} \ddot{v}_{g_{m}}}(\omega)}
$$

where $S_{\ddot{v}_{g_{1}} \ddot{g}_{g_{1}}}(\omega)$ and $S_{\ddot{v}_{g_{m}}}{\ddot{g_{s}}}_{g_{m}}(\omega)$ indicate the autopower spectral density functions of the accelerations at the support points $l$ and $m$, respectively. In the case of homogeneous ground motion for which $S_{\dot{v}_{g_{1}} \ddot{v}_{g_{1}}}(\omega)=S_{\ddot{v}_{g_{m}}} \ddot{v}_{g_{m}}(\omega)=S_{\dot{v}_{g}}(\omega)$, the previous expression can be reduced to

$S_{\ddot{v}_{g_{1}} \ddot{v}_{g_{m}}}(\omega)=\gamma_{1 \mathrm{~m}}(\omega) S_{\ddot{v}_{g}}(\omega)$

where $\gamma_{1 \mathrm{~m}}(\omega)$ is the coherency function and $S_{v_{g}}(\omega)$ is the power spectral density function for uniform surface ground acceleration.

The power spectral density function for ground acceleration is assumed to be of the following form of filtered white noise ground motion model suggested by Clough and Penzien [28]: 
$S_{\ddot{v}_{\mathrm{g}}}(\omega)=\mathrm{S}_{\mathrm{o}}\left(\frac{\omega_{\mathrm{f}}^{4}+4 \xi_{\mathrm{f}}^{2} \omega_{\mathrm{f}}^{2} \omega^{2}}{\left(\omega_{\mathrm{f}}^{2}-\omega^{2}\right)^{2}+4 \xi_{\mathrm{f}}^{2} \omega_{\mathrm{f}}^{2} \omega^{2}}\right)\left(\frac{\omega^{4}}{\left(\omega_{\mathrm{g}}^{2}-\omega^{2}\right)^{2}+4 \xi_{\mathrm{g}}^{2} \omega_{\mathrm{g}}^{2} \omega^{2}}\right)$

in which $\mathrm{S}_{\mathrm{o}}$ is the spectrum of the white-noise bedrock acceleration; $\omega_{\mathrm{f}}$ and $\xi_{\mathrm{f}}$ are the resonant frequency and damping of the first filter, and $\omega_{\mathrm{g}}$ and $\xi_{\mathrm{g}}$ are those quantities of the second filter.

In this study, soft, medium and firm soil types are chosen for the sample. $S_{0}$ is obtained for each soil layer type by equating the variance of the ground acceleration to the variance of Kocaeli Earthquake in 1999. Calculated values of the intensity parameter for each soil type and the filter parameters for these soil types which are proposed Der Kiureghian and Neuenhofer [31] are utilized as shown in Table 1.

The record used is that of the DZC270 component of Duzce, Turkey, Kocaeli Earthquake in 1999, which is given in Fig. 6(a) and lasts for $27.2 \mathrm{sec}$; its power spectral density function, acceleration spectral density function and

$\left|\gamma_{\operatorname{lm}}(\omega)\right|^{\mathrm{i}}=\operatorname{Aexp}\left[-\frac{2 \mathrm{~d}_{\operatorname{lm}}}{\alpha \theta(\omega)}(1-\mathrm{A}+\alpha \mathrm{A})\right]+(1-\mathrm{A}) \exp \left[-\frac{2 \mathrm{~d}_{\operatorname{lm}}}{\theta(\omega)}(1-\mathrm{A}+\alpha \mathrm{A})\right]$

$\theta(\omega)=\mathrm{k}\left[1+\left(\frac{\omega}{2 \pi \mathrm{f}_{\mathrm{o}}}\right)^{\mathrm{b}}\right]^{-0.5}$

where $d_{l m}$ is the distance between support points 1 and $\mathrm{m}$. A, $\alpha, \mathrm{k}, \mathrm{f}_{\mathrm{o}}$ and $\mathrm{b}$ are model parameters.

Table 1. Intensity and filter parameter for different soil types

\begin{tabular}{cccccc}
\hline Type of Soil & $\omega_{\mathrm{f}}(\mathrm{rad} / \mathrm{sn})$ & $\xi_{\mathrm{f}}$ & $\omega_{\mathrm{g}}(\mathrm{rad} / \mathrm{sn})$ & $\xi_{\mathrm{g}}$ & $\mathrm{S}_{\mathrm{o}}\left(\mathrm{m}^{2} / \mathrm{s}^{3}\right)$ \\
\hline Firm & 15.0 & 0.6 & 1.5 & 0.6 & 0.00171 \\
Medium & 10.0 & 0.4 & 1.0 & 0.6 & 0.00255 \\
Soft & 5.0 & 0.2 & 0.5 & 0.6 & 0.00357 \\
\hline
\end{tabular}

displacement spectral density function for different soil types are given Fig. 6(b), Fig. 6(c) and Fig. 6(d), respectively.

The coherency function accounting for the incoherence, wave passage and site-response effects is defined as [31]

$$
\gamma_{\mathrm{lm}}(\omega)=\left|\gamma_{\mathrm{lm}}(\omega)\right|^{\mathrm{i}} \gamma_{\mathrm{lm}}(\omega)^{\mathrm{w}} \gamma_{\mathrm{lm}}(\omega)^{\mathrm{s}}
$$

where $\left|\gamma_{\mathrm{lm}}(\omega)\right|^{\mathrm{i}}$ characterizes the incoherence effect, $\gamma_{\mathrm{lm}}(\omega)^{\mathrm{w}}$ indicates the complex valued wave-passage effect and $\gamma_{\operatorname{lm}}(\omega)^{\mathrm{s}}$ defines the complex valued site-response effect.

In respect of the incoherence effect, the model which is based on the statistical analysis of strong ground motion data and developed by Harichandran and Vanmarcke [32] is 

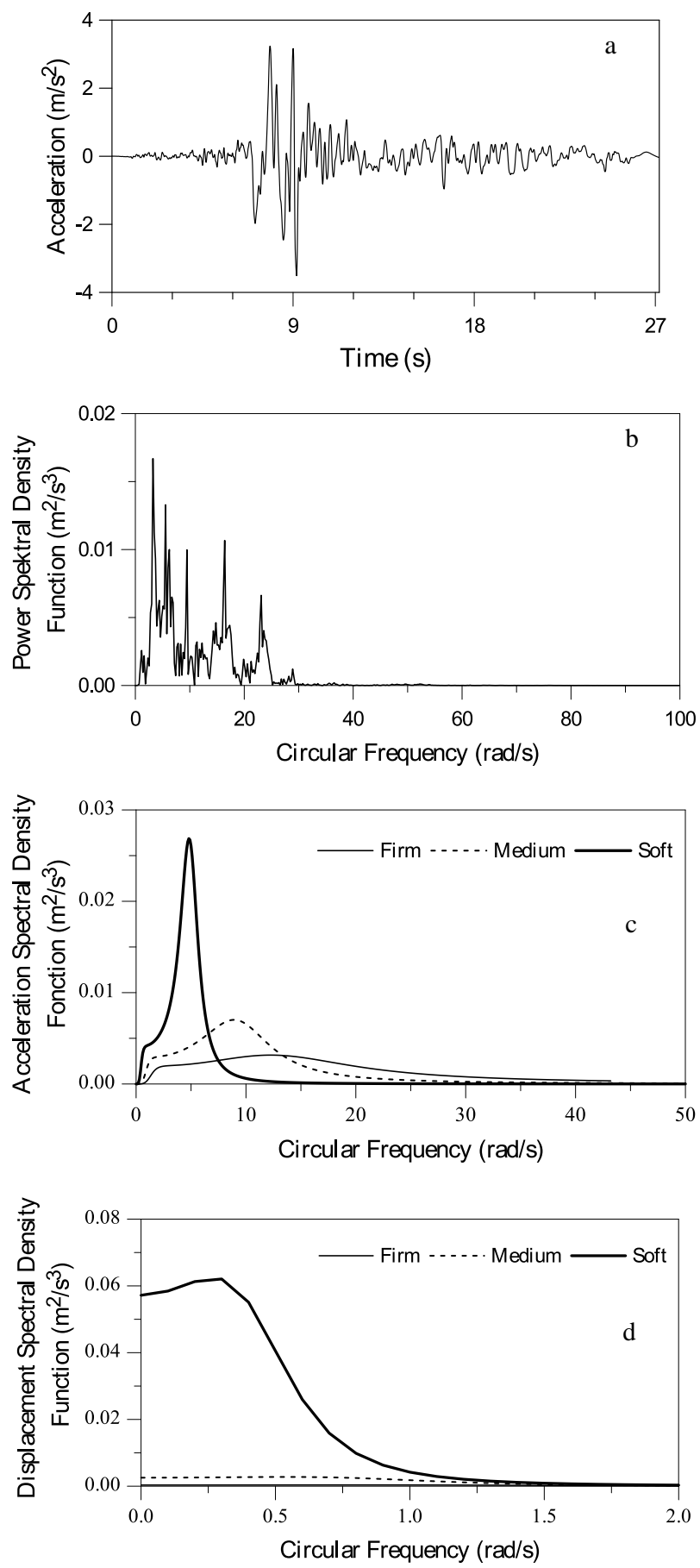

Fig. 6. DZC270 component of the 1999 Kocaeli earthquake; a) Acceleration time history b) Power spectral density function, c) Acceleration spectral density function, d) Displacement spectral density function

In this study, the values obtained by Harichandran et al., [32] were used $(\mathrm{A}=0.636$, $\alpha=0.0186, \mathrm{k}=31200, \mathrm{f}_{\mathrm{o}}=1.51 \mathrm{~Hz}$ and $\left.\mathrm{b}=2.95\right)$.
The wave-passage effect resulting from the difference in the arrival times of waves at support points is defined as 


$$
\gamma_{\mathrm{lm}}(\omega)^{\mathrm{w}}=\exp \left[\mathrm{i}\left(-\omega \mathrm{d}_{1 \mathrm{~m}}^{\mathrm{L}} / \mathrm{v}_{\text {app }}\right)\right]
$$

where $\mathrm{v}_{\text {app }}$ is the apparent wave velocity and $\mathrm{d}_{1 \mathrm{~m}}^{\mathrm{L}}$ is the projection of $\mathrm{d}_{\mathrm{lm}}$ on the ground surface along the direction of propagation of seismic waves [33].

The site-response effect due to the differences in the local soil conditions is obtained as

$$
\gamma_{\mathrm{lm}}(\omega)^{\mathrm{s}}=\exp \left[i \tan ^{-1} \frac{\operatorname{Im}\left[\mathrm{H}_{1}(\omega) \mathrm{H}_{\mathrm{m}}(-\omega)\right]}{\operatorname{Re}\left[\mathrm{H}_{1}(\omega) \mathrm{H}_{\mathrm{m}}(-\omega)\right]}\right]
$$

where $H_{1}(\omega)$ is the local soil frequency response function representing the filtration through soil layers and is defined as [33]:

$$
\mathrm{H}_{1}(\omega)=\frac{\omega_{1}^{2}+2 \mathrm{i} \xi_{1} \omega_{1} \omega}{\omega_{1}^{2}-\omega^{2}+2 \mathrm{i} \xi_{1} \omega_{1} \omega}
$$

in which $\omega_{1}$ and $\xi_{1}$ represent dominant frequency and damping ratio, respectively [31].

\section{Sample Model}

In this study, a single column resting on layered soil medium shown in Fig.7 is considered to compare the results of the finite element and the coupling finite-boundary element models. Width and height of the foundation soil medium considered in the analysis is $8 \mathrm{~m}$ and $4 \mathrm{~m}$, respectively.

The properties of the single column and layered soil medium are given in Table 2 and Table 3, respectively.

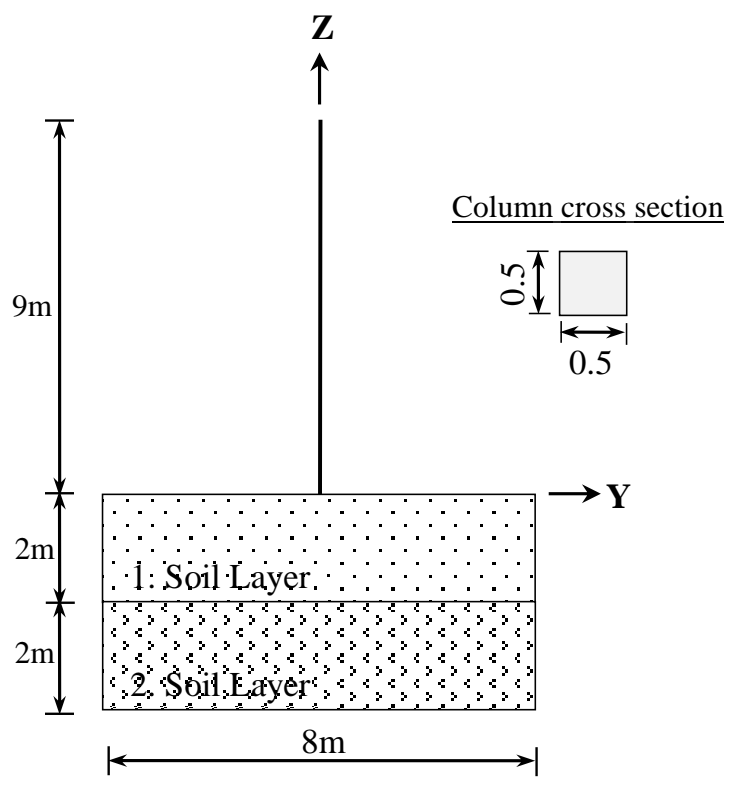

Fig. 7. Selected sample problem

Table 2. The column properties

\begin{tabular}{ccccc}
\hline $\begin{array}{c}\text { Cross sectional area } \\
\mathrm{A}\left(\mathrm{m}^{2}\right)\end{array}$ & $\begin{array}{c}\text { Moment of inertia } \\
\mathrm{I}_{\mathrm{x}-\mathrm{x}}\left(\mathrm{m}^{4}\right)\end{array}$ & $\begin{array}{c}\text { Young's } \\
\text { Modulus } \\
\mathrm{E}\left(\mathrm{kN} / \mathrm{m}^{2}\right)\end{array}$ & $\begin{array}{c}\text { Shear Modulus } \\
\mathrm{G}\left(\mathrm{kN} / \mathrm{m}^{2}\right)\end{array}$ & $\begin{array}{c}\text { Weight per unit } \\
\text { length } \\
\mathrm{W}(\mathrm{kN} / \mathrm{m})\end{array}$ \\
\hline 0.25 & 0.0052 & $32 \times 10^{6}$ & $12.8 \times 10^{6}$ & 6.250 \\
\hline
\end{tabular}


Table 3. The column properties

\begin{tabular}{|c|c|c|c|c|c|c|}
\hline \multirow[t]{2}{*}{ Soil Type } & & $\begin{array}{l}\text { Young's } \\
\text { Modulus, }\end{array}$ & $\begin{array}{c}\text { Shear } \\
\text { Modulus, }\end{array}$ & $\begin{array}{l}\text { Poisson's } \\
\text { Ratio, }\end{array}$ & \multirow[t]{2}{*}{$\rho_{s}\left(\mathrm{kN} / \mathrm{m}^{3}\right)$} & \multirow[t]{2}{*}{$\xi_{s}(\%)$} \\
\hline & & $\mathrm{E}\left(10^{3} \mathrm{kN} / \mathrm{m}^{2}\right)$ & $\mathrm{G}\left(10^{3} \mathrm{kN} / \mathrm{m}^{2}\right)$ & $v$ & & \\
\hline \multirow[t]{2}{*}{ Soft } & Layer\#1 & 224 & 80 & 0.40 & 20 & 0.07 \\
\hline & Layer\#2 & 1540 & 550 & 0.40 & 22 & 0.05 \\
\hline \multirow[t]{2}{*}{ Medium } & Layer\#1 & 1080 & 400 & 0.35 & 20 & 0.04 \\
\hline & Layer\#2 & 7425 & 2750 & 0.35 & 22 & 0.04 \\
\hline \multirow[t]{2}{*}{ Firm } & Layer\#1 & 2430 & 900 & 0.30 & 21 & 0.02 \\
\hline & Layer\#2 & 16900 & 6500 & 0.30 & 25 & 0.02 \\
\hline
\end{tabular}

In the study, to perform the stochastic dynamic finite element analysis of the sample problem the program SVEM [34] developed in FORTRAN is used. In the coupled finite-boundary element model, the program CD2NL [35] is used to obtain equivalent finite element stiffness matrix for the layered soil medium modeled by constant boundary elements. To compare the obtained results the sample problem is separately modeled finite element method and coupled finite-boundary element methods. In the finite element model, a single column and layered soil medium, namely all system, are modeled with finite elements (Fig. 8). In the figure, the column and layered soil medium are modeled with beam and plane elements, respectively. In the coupled finite-boundary element model, the column is modeled with finite elements, whereas layered soil medium is modeled with constant boundary elements as shown in Fig. 9. In the last method, equivalent finite element stiffness matrix concerning layered soil medium is obtained via CD2NL software and this matrix is adapted to the spring element defined in SVEM software as in Fig 9b. In the finite element model whole domain is discretized, whereas in the boundary element model only boundaries of the domain are discretized as shown in Figs. 8 and 9.

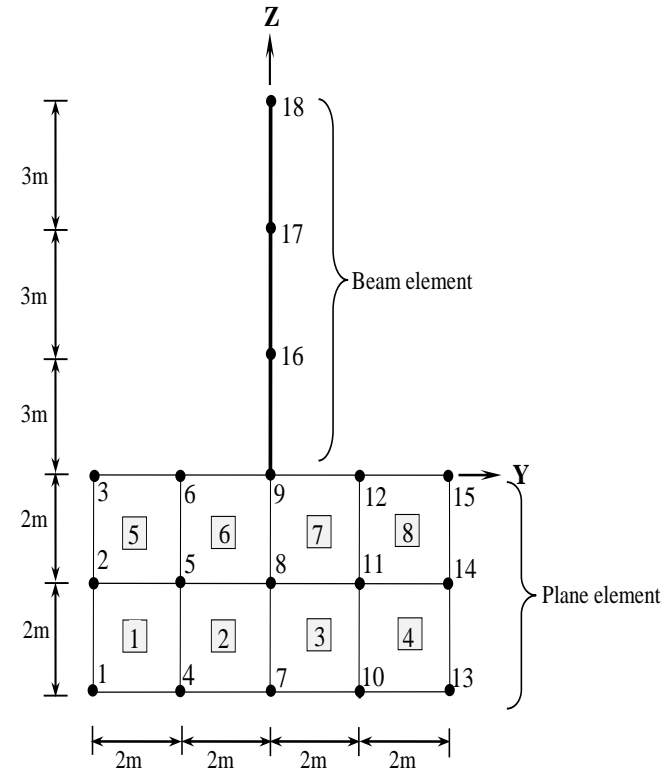

Fig. 8. Finite element model of the problem

Horizontal displacement of the single column resting on soft soil medium is comparatively given in Fig. 10 for the coupling finite-boundary element and the general finite element models concerning the sample system. 


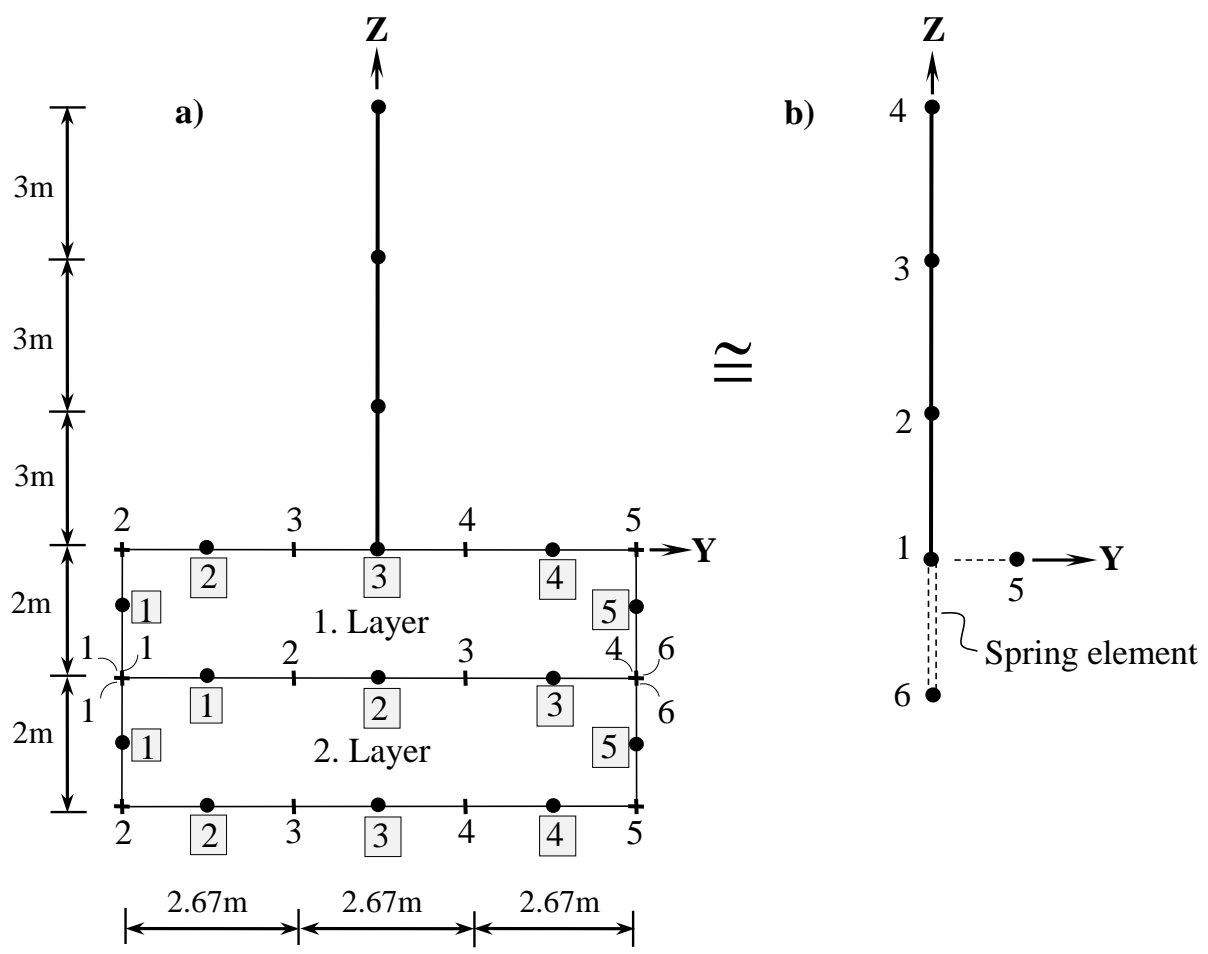

Fig. 9. a) Constant boundary element model of the layered soil medium, b) Coupled finite-boundary element model

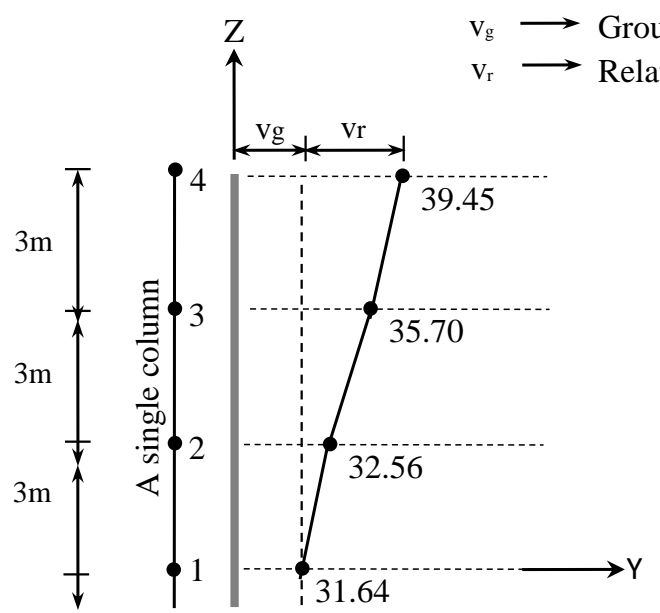

a) The coupling finite-boundary element model

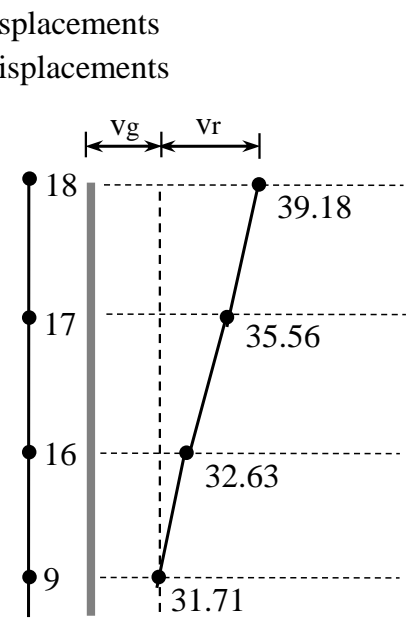

b) Finite element model

Fig. 10. Horizontal displacements of the column supported on soft soil $(\mathrm{cm})$ 
Table 4. Horizontal displacements of the column for each soil type $(\mathrm{cm})$

\begin{tabular}{|c|c|c|c|c|}
\hline \multirow[b]{2}{*}{ Soil Types } & \multirow[b]{2}{*}{ Column height (m) } & \multicolumn{2}{|c|}{ Horizontal displacements $(\mathrm{cm})$} & \multirow[b]{2}{*}{$\begin{array}{c}\text { Difference } \\
(\%)\end{array}$} \\
\hline & & $\begin{array}{c}\text { The coupling finite- } \\
\text { boundary element } \\
\text { model }\end{array}$ & $\begin{array}{l}\text { Finite element } \\
\text { model }\end{array}$ & \\
\hline \multirow{4}{*}{ Soft } & 0 & 31.64 & 31.71 & 0.22 \\
\hline & 3 & 32.56 & 32.63 & 0.23 \\
\hline & 6 & 35.70 & 35.56 & 0.38 \\
\hline & 9 & 39.45 & 39.18 & 0.68 \\
\hline \multirow{4}{*}{ Medium } & 0 & 11.28 & 11.28 & 0.04 \\
\hline & 3 & 11.63 & 11.60 & 0.20 \\
\hline & 6 & 13.37 & 13.25 & 0.93 \\
\hline & 9 & 15.58 & 15.35 & 1.46 \\
\hline \multirow{4}{*}{ Firm } & 0 & 5.50 & 5.49 & 0.02 \\
\hline & 3 & 5.72 & 5.69 & 0.42 \\
\hline & 6 & 6.70 & 6.60 & 1.31 \\
\hline & 9 & 8.16 & 7.98 & 2.21 \\
\hline
\end{tabular}

The records of the DZC270 component of Duzce, Kocaeli Earthquake recorded in 1999 are used and this ground motion is applied in horizontal direction for each model. Horizontal displacements of the single column of each model concerning the sample system are comparatively given for each soil medium in Table 4. Soil properties considered in the analysis is taken from Table 3. It is observed that results obtained of each method are quite close to each other for each soil type.

\section{Conclusion}

The aim of this study is to compare the results of finite element model and the coupling finiteboundary element model when the sample model subjected to spatially varying ground motions including the wave-passage, incoherence and site response effects. The records of Duzce, Kocaeli Earthquake recorded in 1999 are used and these are applied in horizontal direction for each model. The dynamic response of the soil-structure systems is obtained in the frequency domain. In the seismic analysis of the system the substructure method is employed. In the standard finite element model both the structure and layered soil medium are modeled by finite elements, but in the coupling finite-boundary element model the structure and the soil medium are modeled respectively by finite and boundary elements. In the coupling of finiteboundary element method, equivalent finite element approach is used in which boundary element region is transformed as an equivalent finite element and the final system is solved as a stiffness problem. The conclusions drawn from this study can be written as:

- Results of the study show that for all the soil cases considered the coupling finite-boundary element model has shown good agreement with the finite element model and can provide 
realistic and effective modeling of soilstructure interaction problems as compared with standard finite element method results.

- All soil cases considered in the analysis, it is obtained that difference of horizontal displacements increases for both methods as the column height increases and the biggest difference occurs in the top of the column, especially for firm soil case.

- It is shown that the number of elements used in mesh is quite close the coupling finiteboundary element model and classical finite element model for this study. As the size of the problem, number of elements used in mesh rather increases especially finite element model in which is required a mesh of whole domain. Following this study, the number of element and size used in the analysis will compare for both methods.

- It can be concluded that the combination of finite element and boundary element method is an efficient method of analysis especially for soil-structure interaction problems. Therefore, using coupled technique efficient numerical model for soil-structure interaction problems can be treated without compromise in accuracy.

\section{References}

[1] White W, Valliappan S, Lee IK (1977) Unified boundary for finite dynamic models. Journal of Engineering Mechanics ASCE 103 (5):949-964.

[2] Karabalis DL, Beskos DE (1984) Dynamic response of 3-D rigid surface foundations by time domain boundary element method. Earthquake Engineering and Structural Dynamics 12:73-93.

[3] Genes MC, Doganay E, Bikce M, Kacin S (2011) Soil-structure interaction in $\mathrm{rc}$ frame buildings from strong-motion recordings. KSU. Journal of Engineering Sciences 14, 1.

[4] Hughes TJR (1987) The finite element method. New York: Dover Publications INC.

[5] Wolf J, Song C (1996) Finite-element modelling of unbounded media. John Wiley \& Sons, Chi Chester.

[6] Bathe KJ (1996) Finite element procedures. Englewood Cliffs, NJ: Prentice-Hall.
[7] Beskos DE (1987) Boundary element methods in mechanics. Elsevier Science Publishers, Amsterdam, The Netderlands, 598s.

[8] Becker AA (1992) The boundary element method in engineering. Berkshire: McGraw-Hill.

[9] Dominguez J (1993) Boundary elements in dynamics., Southampton and Boston: Computational Mechanics Publications.

[10] Zienkiewicz OC, Kelly DM, Bettes P (1977) The coupling of the finite element method and boundary solution procedures. International Journal of Numerical Methods in Engineering 11:355-376.

[11] Cruse TA, Wilson RB (1978) Advanced application of boundary integral equation methods. Nuclear Engineering and Design 46:223-234.

[12] Brebbia CA, Georgiou P (1979) Combination of boundary and finite elements in elastostatics. Applied Mathematical Modelling 3:212-220.

[13] Brady BHG, Wassyng A (1981) A coupled finite element-boundary element method of stress analysis. International Journal of Rock Mechanics and Mining Sciences 18:475-485.

[14] Tullberg D, Bolteus L (1982) A critical study of different boundary element-stiffness matrices. Boundary Element Methods in Engineering (ed. Brebbia, C. A.), Springer-Verlag, Berlin pp. 621635.

[15] Beer G (1983) BEFE-A combined boundary element finite element computer program. Advances in Engineering Software 6:103-109.

[16] Beer G, Swoboda G (1985) On the efficient analysis of shallow tunnels. Computers and Geotechnics 1:15-31.

[17] Mitsui Y, Ichikawa Y, Obara Y, Kawamoto T (1985) A coupling scheme for boundary and finite elements using a joint element. International Journal Numerical and Analytical Methods in Geomechanics 9:161-172.

[18] Subei N, Saxena SK, Mohammadi J (1991) A BEM-FEM approach for analysis of distresses in pavements. International Journal Numerical and Analytical Methods in Geomechanics 15:103-119.

[19] Yazdchi M, Khalili N, Valliappan S (1999) Dynamic soil-structure interaction analysis via coupled finite-element-boundary-element method. Soil Dynamics and Earthquake Engineering 18:499-517.

[20] Von Estorff O, Firuziaan M (2000) Coupled BEM/FEM approach for nonlinear soil/structure 
interaction. Engineering Analysis with Boundary Elements 24:715-725.

[21] Chouw N, Hao H (2005) Study of SSI and nonuniform ground motion effect on pounding between bridge girders. Soil Dynamics and Earthquake Engineering 25:717-728.

[22] Genes MC, Kocak S (2005) Dynamic soilstructure interaction analysis of layered unbounded media via a coupled finite element/boundary element/scaled boundary finite element model. International Journal for Numerical Methods in Engineering 62:798-823.

[23] Cakir T (2014) Backfill and subsoil interaction effects on seismic behavior of a cantilever wall. Geomechanics and Engineering 6 (2):117-138.

[24] Yaseri A, Bazyar MH, Hataf N (2014) 3D coupled scaled boundary finite-element/finite-element analysis of ground vibrations induced by underground train movement. Computers and Geotechnics 60:1-8

[25] Brebbia CA, Telles JCF, Wrobel LC (1984) Boundary element techniques (Theory and applications in engineering). Springer-Verlag, Berlin.

[26] Dumanoglu AA, Severn RT (1985) Dynamic response of dams and other structures to differential ground motion. Proceedings Institution of Civil Engineers 2(79):429-430.

[27] Dumanoglu AA, Severn RT (1987) Seismic response of modern suspension bridges to asynchronous vertical ground motion. Proceedings Institution of Civil Engineers 83(4):701-730.
[28] Clough RW, Penzien J (1993) Dynamic of structures, Second Edition, McGraw Hill, Inc., Singapure.

[29] Abrahamson NA, Schneider JF, Stepp JC (1991) Empirical spatial coherency functions for application to soil-structure interaction analyses. Earthquake Spectra 7(1):1-27.

[30] Heredia-Zavoni E, Vanmarcke EH (1994) Seismic random vibration analysis of multi-support structural systems. Journal of Engineering Mechanics 120(5):1107-1129.

[31] Der Kiureghian A, Neuenhofer A (1991) A Response spectrum method for multiple-support seismic excitations. Report No. UCB/EERC-91/08, Earthquake Engineering Research Center, College of Engineering, University of California, Berkeley, CA.

[32] Harichandran RS, Vanmarcke EH (1986) Stochastic variation of earthquake ground motion in space and time. Journal of Engineering Mechanics 112(2):154-175.

[33] Der Kiureghian A (1996) A coherency model for spatially varying ground motions. Earthquake Engineering and Structural Dynamics 25(1):99111.

[34] Dumanoglu AA, Soyluk K (2002) SVEM, A stochastic structural analysis program for spatially varying earthquake motions. Turkish Earthquake Foundation, TDV/KT 023-76, Istanbul, Turkey.

[35] Mengi Y, Tanrıkulu AH, Tanrıkulu AK (1994) Boundary element method for elastic media, an Introduction., METU Press, Ankara, Turkey. 